\title{
Article \\ OQPSK Synchronization Parameter Estimation Based on Burst Signal Detection
}

\author{
Zilong Liu $\mathbb{(}$, Kexian Gong, Peng Sun * ${ }^{\mathbb{C}}$, Jicai Deng, Kunheng Zou and Linlin Duan \\ School of Information Engineering, Zhengzhou University, Zhengzhou 450001, China; \\ zilongliu@gs.zzu.edu.cn (Z.L.); kxgong@zzu.edu.cn (K.G.); iejcdeng@zzu.edu.cn (J.D.); \\ zoukunheng@gs.zzu.edu.cn (K.Z.); llduan@126.com (L.D.) \\ * Correspondence: iepengsun@zzu.edu.cn
}

Citation: Liu, Z.; Gong, K.; Sun, P.; Deng, J.; Zou, K.; Duan, L. OQPSK Synchronization Parameter Estimation Based on Burst Signal Detection. Electronics 2021, 10, 69 https://doi.org/10.3390/ electronics10010069

Received: 6 December 2020 Accepted: 29 December 2020 Published: 2 January 2021

Publisher's Note: MDPI stays neutral with regard to jurisdictional clai$\mathrm{ms}$ in published maps and institutional affiliations.

Copyright: (C) 2021 by the authors. Licensee MDPI, Basel, Switzerland. This article is an open access article distributed under the terms and conditions of the Creative Commons Attribution (CC BY) license (https:// creativecommons.org/licenses/by/ $4.0 /)$.
Abstract: The fast estimation of synchronization parameters plays an extremely important role in the demodulation of burst signals. In order to solve the problem of high computational complexity in the implementation of traditional algorithms, a synchronization parameter (frequency offset, phase offset, and timing error) estimation algorithm based on Offset Quadrature Phase Shift Keying (OQPSK) burst signal detection is proposed in this article. We first use the Data-Aided (DA) method to detect where the burst signal begins by taking the segment correlation between the receiving signals and the known local Unique Word (UW). In the sequel, the above results are adopted directly to estimate the synchronization parameters, which is obviously different from the conventional algorithms. In this way, the complexity of the proposed algorithm is greatly reduced, and it is more convenient for hardware implementation. The simulation results show that the proposed algorithm has high accuracy and can track the Modified Cramer-Rao Bound (MCRB) closely.

Keywords: synchronization parameter estimation; burst signal detection; data-aided; OQPSK

\section{Introduction}

The importance of satellite communication is not only reflected in the ordinary communication transmission, but it also has a profound impact on production safety and economic development. With the commercialization of the Fifth-Generation (5G) mobile networks in recent years, the integration of satellite communication and ground 5G has become a new hot topic in the industry. Therefore, satellite communication has ushered in a new round of development upsurge in the world, and the development of its technology has also been paid much attention [1].

As a common communication system in satellites, Time Division Multiple Access (TDMA) shares the transmission medium or network, which is conducive to the transmission of burst data. TDMA works by allowing multiple users to use the same frequency in different time slots, where each user occupies one particular slot. Therefore, TDMA has the advantages of high signal quality, good security, and large system capacity [2].

Offset Quadrature Phase Shift Keying (OQPSK) as a modulation method is widely used in satellite communication because of its constant envelope characteristics, good spectral efficiency, and power efficiency. The OQPSK signal is an improved version of the Quadrature Phase Shift Keying (QPSK) signal, which can be obtained by staggering the code streams of the in-phase component and quadrature component of the QPSK signal by half a symbol period in time, such that its phase can only jump 90 degrees at most.

In satellite communications, due to the burstiness and shortness of the burst signal and the huge influence of synchronization parameters on the performance of signal demodulation [3], it is very important to quickly and accurately capture the starting position of the burst signal and estimate its synchronization parameters. In addition, because of the limitation of hardware resources in practical application, the complexity of the algorithm 
is required to be as low as possible to reduce resource consumption. On the other hand, the accuracy of the algorithm should be guaranteed to meet the actual needs.

\subsection{Related Work}

The improved energy method given in [4] achieves a high detection rate, but it uses the center of the energy window as the starting point of the burst signal, so there is a certain deviation from the true starting point. Cross-correlation is then used in the earliest burst signal detection based on the Data-Aided (DA) method; however, its performance is poor in the presence of frequency offset [5]. The double correlation [6] can effectively eliminate the influence brought by frequency offset. Unfortunately, it contains many conjugate multiplications, resulting in high computational complexity. In [7], a segment correlation algorithm was introduced to greatly reduce the complexity at the expense of part of the anti-frequency offset ability. The burst signal detection adopted in this article is further simplified on the basis of segment correlation.

Once the burst signal detection is done, we need to estimate the synchronization parameters. Synchronization includes carrier synchronization and timing synchronization, in which carrier synchronization includes frequency offset synchronization and phase offset synchronization. We should note that for burst signals, the synchronization parameter estimation often adopts the feedforward algorithm, so the method based on the phase-locked loop [8] is not applicable here. A code-assisted carrier synchronization algorithm was proposed in [9], but this method requires the use of channel coding, and the iterative concept makes its implementation more complicated. In [10], a low-complexity carrier synchronization method was designed under the assumption of a special frame structure. The L\&W, KAY, L\&R, and Fitz [11-14] algorithms are commonly used to feedforward estimate the frequency offset. However, the accuracy and estimation range of these algorithms cannot be well coordinated. More specifically, L\&W and KAY have a wide estimation range, but low accuracy, while L\&R and Fitz have a high accuracy, but a narrow estimation range, and their complexities increase with the improvement of the estimation accuracy. The phase offset estimation is usually performed after the frequency offset is estimated. Finally, the received signal needs to be compensated for the frequency offset based on the above results, and then, the Maximum-Likelihood (ML) principle is applied to estimate the phase offset.

For timing error estimation, the commonly used feedforward algorithms are the Lee, AVN, and Zhu [15-17] algorithms; nevertheless, their performances rely heavily on the roll-off factor, and they cannot be directly applied to OQPSK signals. In [18] (in the simulation, we call the algorithm Ye), a DA-based feedforward timing error estimation algorithm using trigonometric interpolation was proposed, but this algorithm is disabled for anti-frequency offset.

\subsection{Our Contribution}

In this paper, we propose an OQPSK synchronization parameter estimation algorithm based on burst signal detection in satellite communications. The main contributions of this paper are the following:

(1) We exploit a simplified segment correlation algorithm in burst signal detection, where the sequential results of segment correlation are then efficiently used to estimate the synchronization parameters. We should note that there is no correlation between burst the signal detection and synchronization parameter estimation of traditional algorithms. To this degree, the proposed algorithm has significantly low complexity.

(2) For frequency offset estimation, the proposed algorithm can achieve high estimation accuracy and is relatively robust to the estimation range.

(3) For timing error estimation, the proposed algorithm is almost not affected by the roll-off factor, and it also has the ability of anti-frequency offset.

(4) The simulation results show that the algorithm has high accuracy, and its performance is close to the Modified Cramer-Rao Bound (MCRB). 
The rest of this article is arranged as follows: Section 2 is the algorithm description; Section 3 is the performance evaluation; and Section 4 is the conclusions.

\section{Algorithm Description}

The flowchart of this article is shown in Figure 1, where we first detect the burst signal to find the frame header of the received signal. In the sequel, we exploit the above correlation value to estimate the frequency offset, which is compensated for the true carrier frequency and closely followed by the phase offset estimation part. Once again, we apply the interpolation method to the correlation peak in the frame header detection to obtain the timing error.

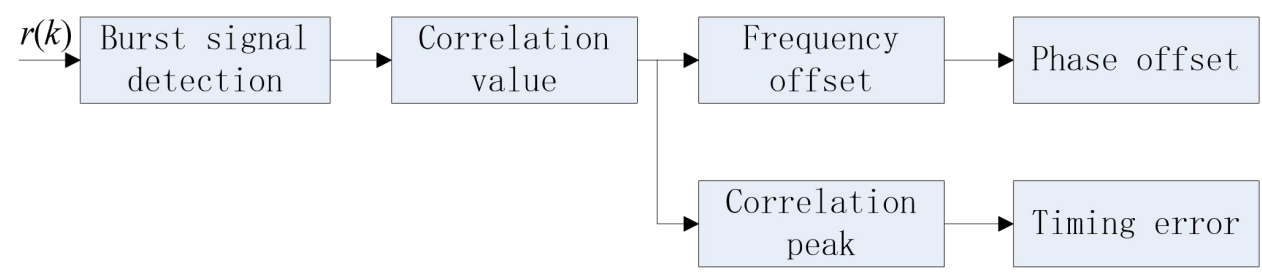

Figure 1. Algorithm flowchart.

\subsection{Burst Signal Detection}

Without considering the timing error, the received signal model can be represented as:

$$
r(k)=\exp \left(j\left(\theta+2 \pi f_{d} k T+\theta_{0}\right)\right)+n(k)
$$

where $e^{j \theta}$ is the $M$-ary phase-modulated symbol, $n(k)$ denotes Additive White Gaussian Noise (AWGN), $T$ stands for the symbol period, and $f_{d}$ and $\theta_{0}$ represent the frequency offset and phase offset, respectively. Applying double correlation between $r(k)$ in Equation (1) and the known local Unique Word (UW) $c(k)$ yields:

$$
L_{0}(v)=\sum_{i=1}^{N-1}\left\{\left|\sum_{k=i}^{N-1} r(v+k) c(k)^{*} r^{*}(v+k-i) c(k-i)\right|-\sum_{k=v+i}^{v+N-1}|r(k)||r(k-i)|\right\}
$$

where $v$ is the moment of judgment. In Equation (2), $\sum_{k=i}^{N-1} r(v+k) c(k)^{*} r^{*}(v+k-i) c(k-i)$ is the double correlation term, and $\sum_{k=v+i}^{v+N-1}|r(k)||r(k-i)|$ is the random data correction term. Usually, the random data correction term can be omitted [6], such that Equation (2) can be approximated as:

$$
L_{1}(v)=\sum_{i=1}^{N-1}\left\{\left|\sum_{k=i}^{N-1} r(v+k) c(k)^{*} r^{*}(v+k-i) c(k-i)\right|\right\}
$$

The reason we chose double correlation in Equations (2) and (3) is that cross-correlation is dysfunctional when frequency offset exists. Unfortunately, this brings unacceptable complexity and makes its implementation in hardware impossible. In order to reduce the computational complexity, the double correlation method can be improved by the concept of segments [7]; that is:

$$
\begin{gathered}
x_{i}=\sum_{k=0}^{L-1} r(v+k+i L) c(k+i L)^{*} \\
L_{2}(v)=\sum_{j=1}^{N / L-1}\left|\sum_{i=j}^{N / L-1} x_{i} x_{i-j}^{*}\right|
\end{gathered}
$$

where $N$ and $L$ are the lengths of the local $U W$ and each segment of $U W$, respectively. The segment correlation takes the conjugate multiplication between different segments to eliminate the influence of frequency offset. It can be seen from Equations (4) and (5) that the complexity of $L_{2}(\mu)$ is greatly reduced compared to that of $L_{1}(\mu)$. 
Normally, the received signal should be up-sampled, but when the local $U W$ is correlated with the received signal, we can choose whether the local $U W$ is up-sampled or not. In the simulation, we find that using local $U W$ without up-sampling in QPSK modulation yields acceptable performance, but this does not work for OQPSK modulation. This is because OQPSK staggers the in-phase component and quadrature component by half a symbol period, and the local $U W$ will lose the information of the half symbol if the local $U W$ is not up-sampled. Obviously, the solution to that is to up-sample the local $U W$ and stagger its in-phase component and quadrature component by half a symbol period.

We find it a convenient approximation of Equation (5) to only consider the case of $j=1$. This is because the correlation of the segment correlation algorithm is mainly dominated by the adjacent segments ( $j=1$ case). Therefore, Equation (5) can be written as follows:

$$
L_{3}(v)=\left|\sum_{i=1}^{N / L-1} x_{i} x_{i-1}^{*}\right|
$$

Undoubtedly, $L_{3}(v)$ has lower complexity, but results in extra performance loss. Fortunately, our simulation shows that this loss is acceptable. More in detail, the complexity comparison between $L_{1}(v), L_{2}(v)$, and $L_{3}(v)$ is explained in Table 1.

Table 1. Algorithmic complexity comparison of burst signal detection.

\begin{tabular}{ccc}
\hline & Multiplication & Addition \\
\hline$L_{1}(v)$ & $6 N^{2}-6 N$ & $N^{2}-2 N$ \\
$L_{2}(v)$ & $2(N / L)^{2}+4 N-2 N / L$ & $(N / L)^{2}+2 N-4 N / L$ \\
$L_{3}(v)$ & $4 N+4 N / L-4$ & $2 N-4$ \\
\hline
\end{tabular}

\subsection{Synchronization Parameter Estimation}

Once the frame header of the burst signal is detected, the following work will be the synchronization parameter (frequency offset, phase offset, and timing error) estimation.

\subsubsection{Frequency Offset Estimation}

We start our derivation of the estimation of frequency offset in Equation (4). Specifically, the term:

$$
\begin{aligned}
r(k) c^{*}(k) & =\exp \left(j\left(2 \pi f_{d} k T+\theta_{0}\right)\right)+n(k) c^{*}(k) \\
& =\exp \left(j\left(2 \pi f_{d} k T+\theta_{0}\right)\right)+n^{\prime}(k)
\end{aligned}
$$

In order to facilitate the derivation, we temporarily ignore the influence of the noise term $n^{\prime}(k)$ and define $f^{\prime} \triangleq 2 \pi f_{d} T$. Equation (7) can be written as follows:

$$
r(k) c^{*}(k)=\exp \left(j\left(f^{\prime} k+\theta_{0}\right)\right)
$$

As we mentioned earlier, the value of $v$ indicates the judgment time, which only represents the starting position of the burst signal and does not affect the parameter estimation. Thus, we can set take the value of $v$ to zero. Then, incorporating Equation (8) into Equation (4):

$$
\begin{gathered}
x_{i}=\exp \left(j\left(f^{\prime} i L+\theta_{0}\right)\right)\left\{1+\exp \left(j f^{\prime}\right)+\ldots+\exp \left(j(L-1) f^{\prime}\right)\right\} \\
x_{i-1}=\exp \left(j\left(f^{\prime}(i-1) L+\theta_{0}\right)\right)\left\{1+\exp \left(j f^{\prime}\right)+\ldots+\exp \left(j(L-1) f^{\prime}\right)\right\}
\end{gathered}
$$

In order to obtain $L_{3}(v)$ in Equation (6), we first simplify $x_{i} x_{i-1}^{*}$ :

$$
x_{i} x_{i-1}^{*}=A \exp \left(j f^{\prime} L\right)
$$


where:

$$
A=L+2(L-1) \cos f^{\prime}+2(L-2) \cos 2 f^{\prime}+\ldots+2 \cos (L-1) f^{\prime}
$$

Equation (12) implies that the value of $A$ is a real number, and accordingly, it will not affect the result of the complex angle of Equation (11). Obviously, the following results can be obtained:

$$
f^{\prime}=\frac{1}{L} \arg \left\{x_{i} x_{i-1}^{*}\right\}
$$

The result of Equation (13) is in an ideal case, that is no noise is considered. Unfortunately, noise has a non-negligible effect on the frequency offset estimation. In order to improve the performance of the estimation, we accumulate the value of $x_{i} x_{i-1}^{*}$ to smooth the effect of noise. We should also note that in order to facilitate the derivation, we defined $f^{\prime} \triangleq 2 \pi f_{d} T$ above. Consequently, the final frequency offset can be calculated as:

$$
\hat{f}_{d}=\frac{1}{2 \pi L} \arg \left\{\sum_{i=1}^{N / L-1} x_{i} x_{i-1}^{*}\right\}
$$

Equation (14) indicates that the frequency offset estimation range is inversely proportional to $L$, and its estimation accuracy is directly proportional to it. It is particularly important to note that $\sum_{i=1}^{N / L-1} x_{i} x_{i-1}^{*}$ in Equation (14) equals Equation (6) after removing the modulus. Therefore, the burst signal detection and parameter estimation algorithm are connected here. In this case, we can directly use the results of burst signal detection to estimate the frequency offset, which is why the complexity of the algorithm in this article is greatly reduced. Table 2 is a comparison of the complexity of the frequency offset estimation algorithms. $N_{0}$ in L\&R and Fitz is usually $N / 2$.

Table 2. Comparison of the complexity of frequency offset estimation algorithms.

\begin{tabular}{cccc}
\hline & Multiplication & Addition & Complex Angle \\
\hline L\&R & $12 N N_{0}-6 N_{0}^{2}-6 N_{0}$ & $\left(2 N-N_{0}-1\right)\left(N_{0}-1\right)$ & 1 \\
Fitz & $12 N N_{0}-6 N_{0}^{2}-6 N_{0}$ & $\left(2 N-N_{0}-1\right)\left(N_{0}-1\right)$ & $N_{0}$ \\
Kay & $14(N-1)$ & $2(N-1)$ & 1 \\
L\&W & $14(N-1)$ & $2(N-1)$ & 1 \\
This article & 1 & 0 & 1 \\
\hline
\end{tabular}

\subsubsection{Phase Offset Estimation}

We perform phase offset estimation after frequency offset estimation is completed. The first step of phase offset estimation is to compensate for the frequency offset in the frame header:

$$
\begin{aligned}
y(k) & =r(k) * \exp \left(-2 \pi \hat{f}_{d} k T\right) \\
& =\left\{\exp \left(j\left(\theta+2 \pi f_{d} k T+\theta_{0}\right)\right)+n(k)\right\} * \exp \left(-j 2 \pi \hat{f}_{d} k T\right) \\
& =\exp \left(j\left(\theta+\theta_{0}\right)\right)+n^{\prime \prime}(k)
\end{aligned}
$$

where $y(k)$ is the frame header after removing the frequency offset and $n^{\prime \prime}(k)=n(k) *$ $\exp \left(-j 2 \pi \hat{f}_{d} k T\right)$ is the noise item. According to the principle of ML, the DA phase offset estimation algorithm can be obtained as [19]:

$$
\hat{\theta}_{0}=\arg \left\{\sum_{k=0}^{N-1} y(k) c^{*}(k)\right\}
$$




\subsubsection{Timing Error Estimation}

After the burst signal detection is completed, in addition to estimating the above carrier synchronization parameters, it is also necessary to estimate the timing error. The signal model with timing error is:

$$
r(t)=e^{j \theta_{0}} \sum_{l} a(l) g(t-l T-\tilde{\tau})+n(t)
$$

where $\{a(l)\}$ represents the independent and identically distributed information sequence and $g(t)$ and $\tau$ denote the baseband shaping function and timing error, respectively. When the received signal is sampled at $t=k T+\tilde{\tau}$, the sampled data $r(k T+\tilde{\tau})$ can be obtained. The ML-based data-aided timing error estimation formula given in [20] is as follows:

$$
\Lambda(\tilde{\tau})=\left|\sum_{k=0}^{N-1} \hat{c}^{*}(k) r(k T+\tilde{\tau})\right|^{2}
$$

If removing the square, Equation (18) is accurate for the burst signal detection based on cross-correlation. The process of capturing the frame header is the process of searching for correlation peaks. When Equation (18) achieves the maximum value, the timing error can be calculated as:

$$
\tilde{\tau}=\underset{\tilde{\tau}}{\operatorname{argmax}}\left\{\left|\sum_{k=0}^{N-1} \hat{c}^{*}(k) r(k T+\tilde{\tau})\right|^{2}\right\}
$$

As mentioned above, the cross-correlation algorithm has the fatal disadvantage of no anti-frequency offset. Fortunately, we found that the segment correlation used in this article is also the process of capturing the frame header, and its maximum value position is the same as Equation (18), that is when the local $U W$ is completely aligned with the $U W$ in the received signal. Therefore, the cross-correlation peak in Equation (19) can be replaced by the segment correlation peak in Equation (6), and the timing error estimation algorithm at this time obtains the ability of anti-frequency offset. Thus, burst signal detection and timing error estimation are linked via:

$$
\tilde{\tau}=\underset{\tilde{\tau}}{\operatorname{argmax}}\left\{\left|\sum_{i=1}^{N / L-1} x_{i} x_{i-1}^{*}\right|^{2}\right\}
$$

The interpolation method is usually adopted to calculate the maximum value of Equation (20); here, we chose a simple and highly accurate trigonometric interpolation method [21]. It should be noted that the number of points used must be an even number. In this article, there were four points employed for interpolation, and each symbol took two points; thus, a total of two symbols were required. The four-point interpolation formula given in [21] is as follows:

$$
z(\mu)=\frac{1}{4} \operatorname{Re}\left(C_{0}+2 C_{1} e^{j \pi \mu / 2}+C_{2} e^{j \pi \mu}\right)
$$

Among them:

$$
\begin{aligned}
& C_{0}=z(-1)+z(0)+z(1)+z(2) \\
& C_{1}=[z(0)-z(2)]+j[-z(1)+z(-1)] \\
& C_{2}=z(0)-z(1)+z(2)-z(-1)
\end{aligned}
$$

To find the timing error, we only need to find out what the value of $\mu$ is to maxi$\operatorname{mize} z(\mu)$ :

$$
\hat{\mu}=\arg \left\{\max _{\mu}\left|\operatorname{Re}\left(C_{0}+2 C_{1} e^{j \pi \mu / 2}+C_{2} e^{j \pi \mu}\right)\right|^{2}\right\}
$$


Therefore, finding the timing error is transformed into the problem of finding the maximum value of $\operatorname{Re}\left(C_{0}+2 C_{1} e^{j \pi \mu / 2}+C_{2} e^{j \pi \mu}\right)$. We define:

$$
Z(\mu) \triangleq \operatorname{Re}\left(C_{0}+2 C_{1} e^{j \pi \mu / 2}+C_{2} e^{j \pi \mu}\right)
$$

For the convenience of calculation, we define $C_{1} \triangleq A+B j$, where $A=z(0)-z(2)$, $B=-z(1)+z(-1)$. According to the Euler formula, $e^{j \pi \mu / 2}=\cos \frac{\pi \mu}{2}+j \sin \frac{\pi \mu}{2}, e^{j \pi \mu}=$ $\cos \pi \mu+j \sin \pi \mu$ can be obtained; thus, Equation (24) can be rewritten as:

$$
Z(\mu)=2 A \cos \frac{\pi \mu}{2}-2 B \sin \frac{\pi \mu}{2}+C_{2} \cos \pi \mu
$$

The derivative of the above equation:

$$
Z^{\prime}(\mu)=-A \pi \sin \frac{\pi \mu}{2}-B \pi \cos \frac{\pi \mu}{2}-C_{2} \pi \sin \pi \mu
$$

Take zero for the above equation, then solve it; we can obtain:

$$
\hat{\mu}=-\frac{2}{\pi} \arctan \left(\frac{2 C_{2}}{A} \sin \frac{\pi \hat{\mu}}{2}+\frac{B}{A}\right)
$$

According to Equation (27), the corresponding timing error can be calculated as:

$$
\hat{\tau}=\frac{\hat{\mu} T}{P}=-\frac{T}{\pi} \arctan \left(\frac{2 C_{2}}{A} \sin \pi \hat{\tau}+\frac{B}{A}\right)
$$

where $P$ is the number of sampling points used for each symbol during interpolation, and $P=2$ in this article. The value of $\tau$ cannot be calculated by Equation (28) because the equation is already in its simplest form; thus, we have to do approximate processing. The four points adopted in the interpolation are approximately symmetrical, in which the values of $z(0)$ and $z(1)$ in the middle are larger and the values of $z(-1)$ and $z(2)$ on both sides are smaller. Therefore, we can get a larger $A$ and a smaller $C_{2}$, and further, $\frac{2 C_{2}}{A} \sin \pi \hat{\tau}$ can be ignored:

$$
\hat{\tau}=-\frac{T}{\pi} \arctan \frac{B}{A}=-\frac{T}{\pi} \arctan \frac{-z(1)+z(-1)}{z(0)-z(2)}
$$

Although we obtain Equation (29) by ignoring $\frac{2 C_{2}}{A} \sin \pi \hat{\tau}$, simulation indicates that it still reaches high accuracy.

\section{Performance Evaluation}

\subsection{Performance Simulation of Burst Signal Detection}

Tables 3 and 4 are the comparisons of the false alarm rate and detection rate of OQPSK burst signal detection under local $U W$ being up-sampled four times and not up-sampled in the case of Equation (5). Simulation conditions: in the case of being up-sampled, the added normalized frequency offset is 0.04 (relative to the symbol rate and the same below); the total length of $U W$ after being up-sampled four times is $N=108$; the number of segments is $M=9$; and the local $U W$ length of each segment is $L=N / M=12$; in the case of not being up-sampled, the added normalized frequency offset is $0.04, N=27, M=9$, and $L=N / M=3$. We performed 10,000 simulations. From Tables 3 and 4 , it can be seen that the performance of the local $U W$ when it is up-sampled four times is much better than not being up-sampled. This is because there are more points after being up-sampled, so the value of the correlation result is larger, but the more important reason is that the OQPSK signal can stagger its in-phase component and quadrature component by half a symbol period only after being up-sampled; otherwise, the partial correlation will be lost. 
Table 3. Comparison of the false alarm rate between the local Unique Word $(U W)$ being up-sampled and not being up-sampled.

\begin{tabular}{|c|c|c|c|c|c|c|}
\hline Local $u W \quad$ SNR & $0 \mathrm{~dB}$ & $1 \mathrm{~dB}$ & $2 \mathrm{~dB}$ & $3 \mathrm{~dB}$ & $4 \mathrm{~dB}$ & $5 \mathrm{~dB}$ \\
\hline up-sampled & $1.0 \times 10^{-5}$ & $3.7 \times 10^{-6}$ & $7.9 \times 10^{-7}$ & $2.2 \times 10^{-7}$ & 0 & 0 \\
\hline not up-sampled & $2.7 \times 10^{-4}$ & $2.1 \times 10^{-4}$ & $1.7 \times 10^{-4}$ & $1.3 \times 10^{-4}$ & $1.3 \times 10^{-4}$ & $9.2 \times 10^{-5}$ \\
\hline
\end{tabular}

Table 4. Comparison of the detection rate between the local $U W$ being up-sampled and not being up-sampled.

\begin{tabular}{|c|c|c|c|c|c|c|}
\hline Local UW & $0 \mathrm{~dB}$ & $1 \mathrm{~dB}$ & $2 \mathrm{~dB}$ & $3 \mathrm{~dB}$ & $4 \mathrm{~dB}$ & $5 \mathrm{~dB}$ \\
\hline up-sampling & $99.03 \%$ & $99.67 \%$ & $99.993 \%$ & $99.998 \%$ & $100 \%$ & $100 \%$ \\
\hline not up-sampling & $77.25 \%$ & $85.51 \%$ & $91.77 \%$ & $95.67 \%$ & $98.3 \%$ & $99.27 \%$ \\
\hline
\end{tabular}

Tables 5 and 6 are the comparisons of the false alarm rate and detection rate, respectively, of the segment correlation algorithm before and after the simplification. Simulation conditions: the added normalized frequency offset is $0.04, N=108, M=9$, and $L=12$. We performed 10,000 simulations. Tables 5 and 6 indicate that the performance of the simplified segment correlation algorithm is slightly lower than that before the simplification, but the decrease is not significant. This is because the correlation of the segment correlation algorithm is mainly concentrated between adjacent segments. When $j=1$, the correlation is maximum, and the correlation decreases with the increase of $j$. Therefore, this paper adopts the simplified algorithm, that is the case where the value of $j$ is only one, which can be exchanged for a further reduction in the amount of calculation at the cost of a small performance loss.

Table 5. Comparison of the false alarm rate between $L_{2}(\mu)$ and $L_{3}(\mu)$.

\begin{tabular}{|c|c|c|c|c|c|c|}
\hline$\underbrace{\text { SNR }}_{j \text { Value }}$ & $0 \mathrm{~dB}$ & $1 \mathrm{~dB}$ & $2 \mathrm{~dB}$ & $3 \mathrm{~dB}$ & $4 \mathrm{~dB}$ & $5 \mathrm{~dB}$ \\
\hline $1: N-1$ & $1.0 \times 10^{-5}$ & $3.7 \times 10^{-6}$ & $7.9 \times 10^{-7}$ & $2.2 \times 10^{-7}$ & 0 & 0 \\
\hline 1 & $1.5 \times 10^{-5}$ & $7.1 \times 10^{-6}$ & $1.6 \times 10^{-6}$ & $5.1 \times 10^{-7}$ & $2.2 \times 10^{-7}$ & 0 \\
\hline
\end{tabular}

Table 6. Comparison of the detection rate between $L_{2}(\mu)$ and $L_{3}(\mu)$.

\begin{tabular}{|c|c|c|c|c|c|c|}
\hline${ }_{j \text { Value }}^{\text {SNR }}$ & $0 \mathrm{~dB}$ & $1 \mathrm{~dB}$ & $2 \mathrm{~dB}$ & $3 \mathrm{~dB}$ & $4 \mathrm{~dB}$ & $5 \mathrm{~dB}$ \\
\hline $1: N-1$ & $99.03 \%$ & $99.67 \%$ & $99.993 \%$ & $99.998 \%$ & $100 \%$ & $100 \%$ \\
\hline 1 & $98.69 \%$ & $99.4 \%$ & $99.86 \%$ & $99.995 \%$ & $99.998 \%$ & $100 \%$ \\
\hline
\end{tabular}

To sum up, for the burst signal detection algorithm, on the one hand, due to the characteristics of the OQPSK signal, the local UW must be up-sampled to ensure its detection performance. On the other hand, in order to reduce the complexity of the algorithm, we further simplify the segment correlation algorithm. Of course, the performance of the algorithm will decrease after simplification, but fortunately, the decrease is not obvious. 


\subsection{Performance Simulation of Synchronization Parameter Estimation}

\subsubsection{Performance Simulation of Frequency Offset Estimation}

Figure 2 shows the Mean Squared Error (MSE) comparison of different frequency offset estimation algorithms. Simulation conditions: the added normalized frequency offset is $0.02 ; N=108$; and the segment length $L$ of the algorithm in this article is $18,12,9$, and 6, respectively. The figure implies that the $L \& R$ and Fitz algorithms have the highest estimation accuracy, and their performance is very close to the MCRB, while the L\&W algorithm has the worst performance. The Kay algorithm only gradually gets better in the case of a high SNR. The performance of the algorithm in this article is proportional to the length of $L$. We can see that when $L=18$, the accuracy of the algorithm in this article is very close to that of the $L \& R$ and Fitz algorithms and is far better than the $L \& W$ algorithm. In addition, when the SNR is lower than $12 \mathrm{~dB}$, its performance is also better than the KAY algorithm.

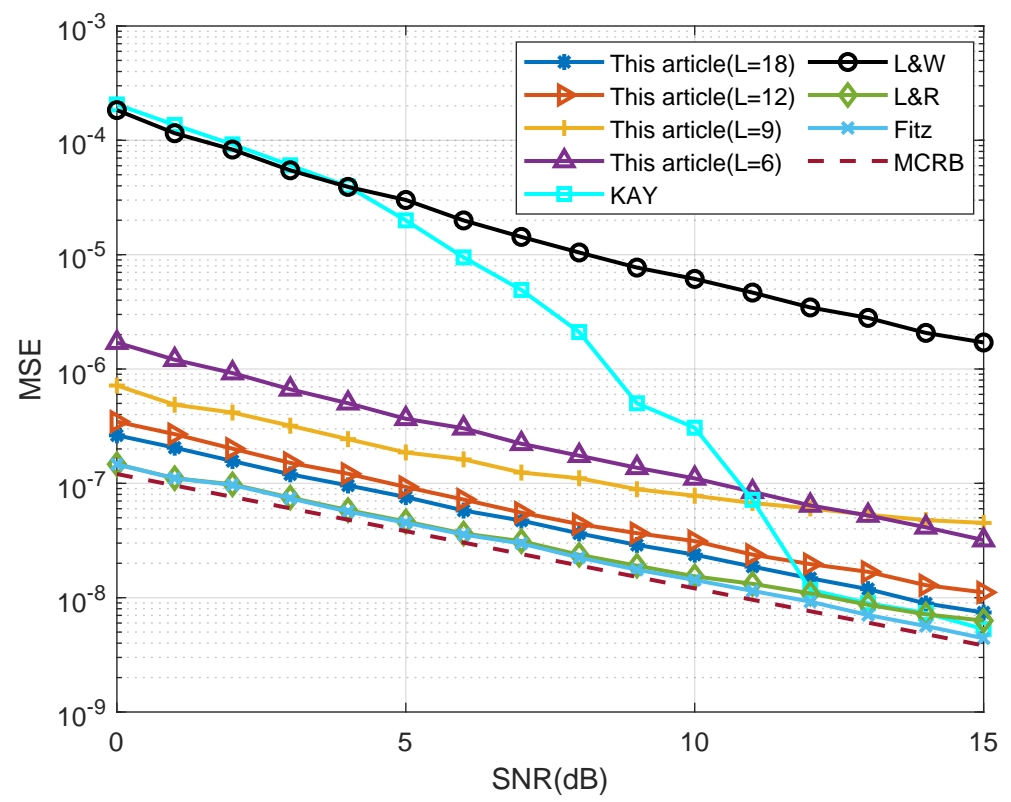

Figure 2. Accuracy of different frequency offset estimation algorithms. MCRB, Modified Cramer-Rao Bound.

Figure 3 shows the comparison of the estimation range of the algorithm in this article and several frequency offset algorithms with high accuracy. Simulation conditions: the added normalized frequency offset is [-0.4:0.4], and its step size is $0.001 ; N=108$; and the segment length $L$ of the algorithm in this article is $18,12,9$, and 6 , respectively. It can be seen from the figure that under the current simulation conditions, the estimation ranges of Fitz and L\&R are (-0.035:0.035) and (-0.07:0.07), respectively. In practical applications, the range of the estimated frequency offset should be at least (-0.1:0.1); thus, the L\&R and Fitz algorithms become invalid in the presence of large frequency offset. While the estimation range of the algorithm in this article can still reach $(-0.11,0.11)$ even in the case of the highest accuracy $(L=18)$. In the following simulations, we select $L=12$, since the $L=12$ case is more robust to frequency offset $(-0.16: 0.16)$, and there is not much difference in the MSE performance between $L=12$ and $L=18$.

In short, as long as the appropriate $L$ is selected, the frequency offset estimation algorithm in this paper not only has an accuracy close to the L\&R and Fitz algorithms, but it also has a much larger estimation range. In addition, the frequency offset estimation algorithm is calculated based on the correlation results generated during the burst signal detection process, so its complexity drops significantly. 


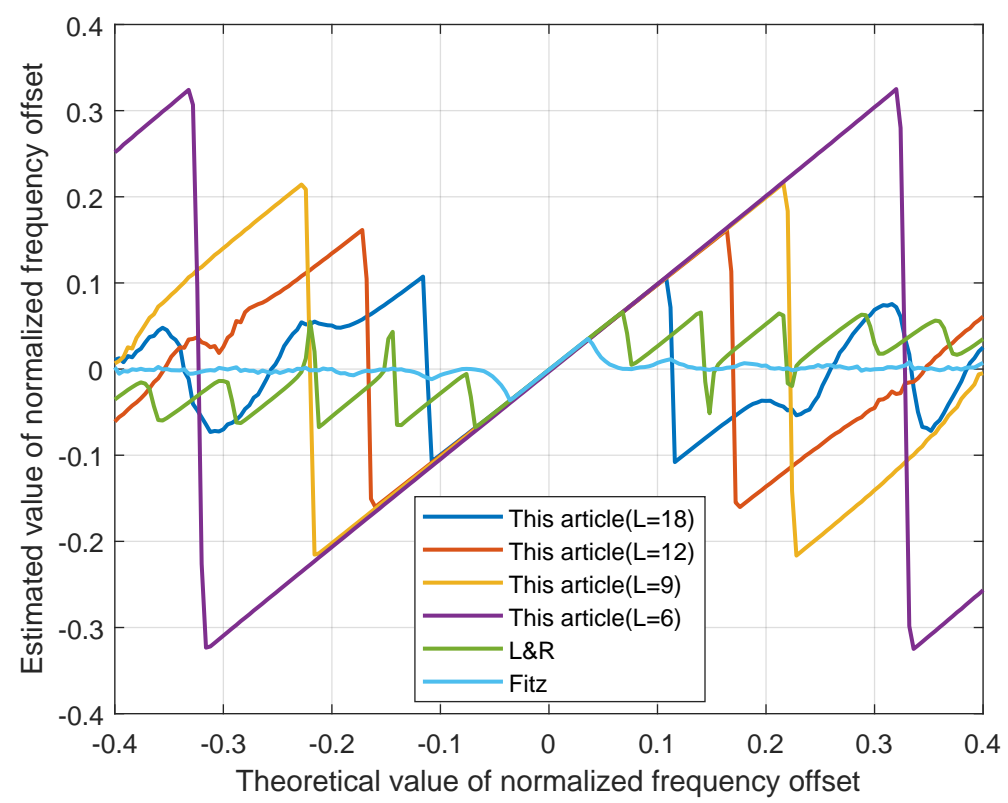

Figure 3. Estimation range of different frequency offset estimation algorithms.

\subsubsection{Performance Simulation of Phase Offset Estimation}

Figure 4 shows the simulation of phase offset estimation accuracy. Simulation conditions: $N=108$; the added phase offsets are $-\frac{3}{4} \pi,-\frac{1}{2} \pi,-\frac{1}{4} \pi, 0, \frac{1}{4} \pi, \frac{1}{2} \pi$, and $\frac{3}{4} \pi$. From the figure, we can see that the MSE of the algorithm closely tracks the MCRB, so its performance is excellent.

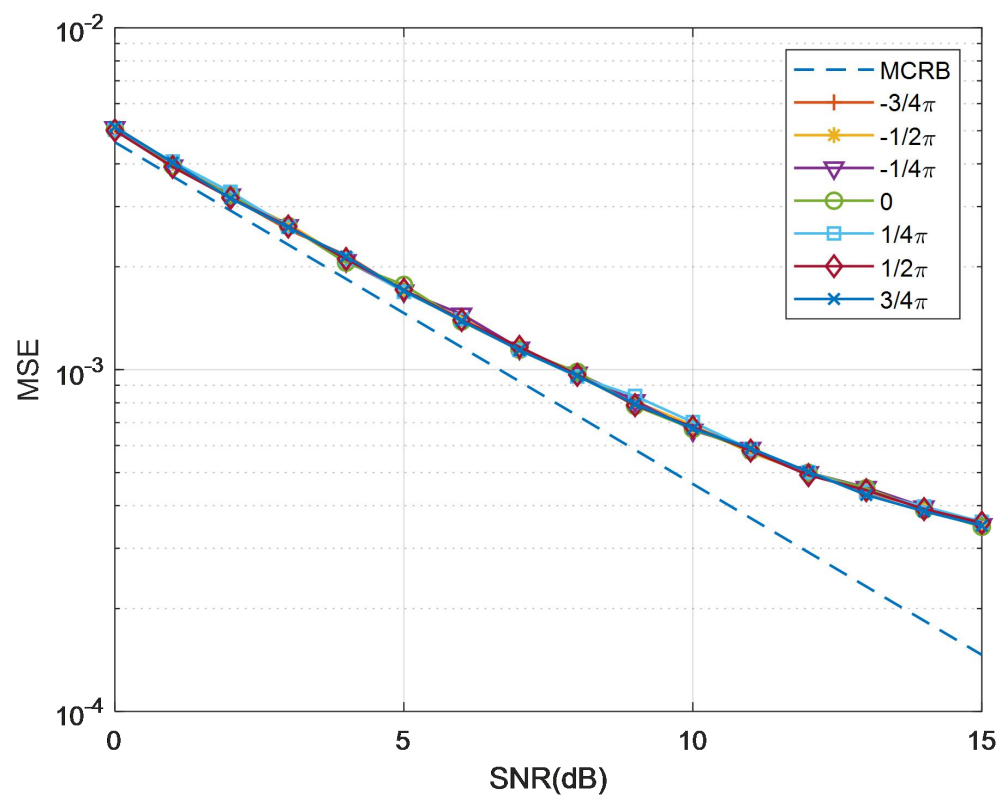

Figure 4. Accuracy of the phase offset estimation algorithm.

Figure 5 shows the effect of different $U W$ lengths on the phase offset estimation performance. Simulation conditions: the added phase offset is $\frac{1}{4} \pi$, and the $U W$ lengths used are $N(N=108), 3 N / 4, N / 2$, and $N / 4$. From the figure, we can see that the performance of the algorithm is proportional to the local UW length used.

In summary, the ML-based phase offset estimation algorithm has better estimation performance, and its performance is directly proportional to the $U W$ length. In actual projects, as long as the performance can meet the needs, the length of the local $U W$ used can be appropriately reduced. For example, when the length of the local UW used in this 
article takes $N / 2$, its accuracy still meets the requirements, and its complexity is reduced by half.

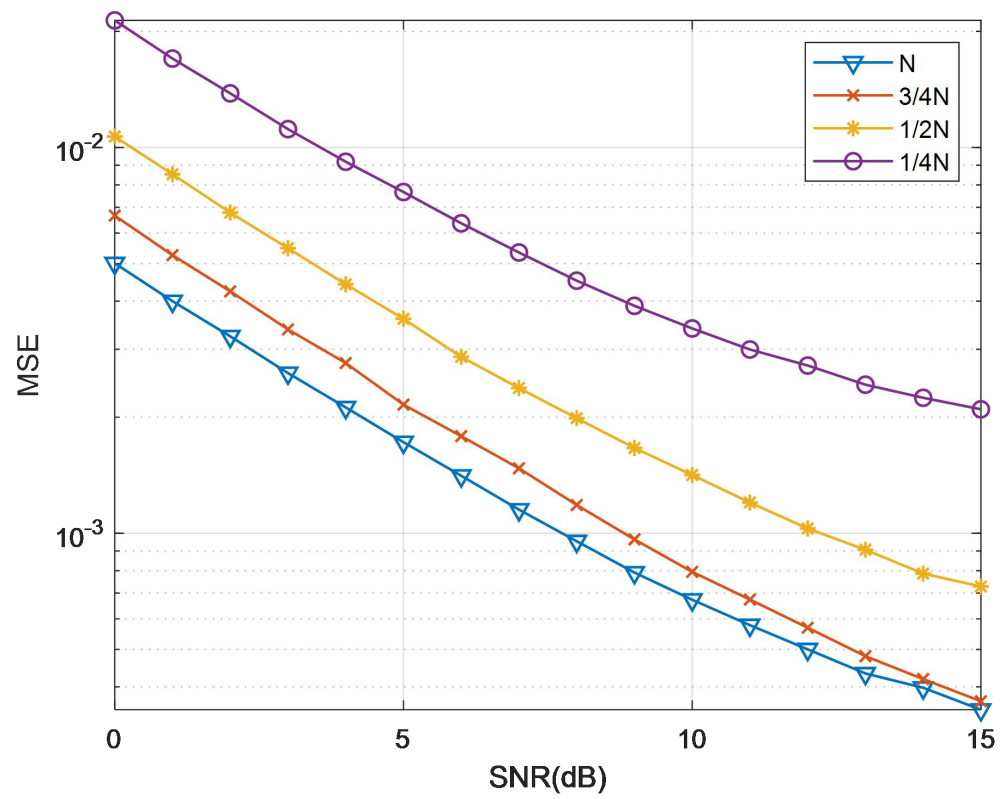

Figure 5. Accuracy of the phase offset estimation algorithm under different $U W$ lengths.

\subsubsection{Performance Simulation of Timing Error Estimation}

Figure 6 shows the estimation accuracy of OQPSK signals under different timing estimation algorithms. Simulation conditions: the roll-off factor is set to $0.35, N=108$; $L=12$; and the frequency offset is zero. It can be seen from the figure that the Lee, Wang, and AVN [15-17] algorithms are completely invalid at this time, and only the suggested algorithm and the Ye algorithm [18] can be used. We note that to make the system suitable for the proposed algorithm and the Ye algorithm, we especially emphasize the up-sampling of local $U W$ and staggering the in-phase component and quadrature component by half a symbol period in Section 2.1.

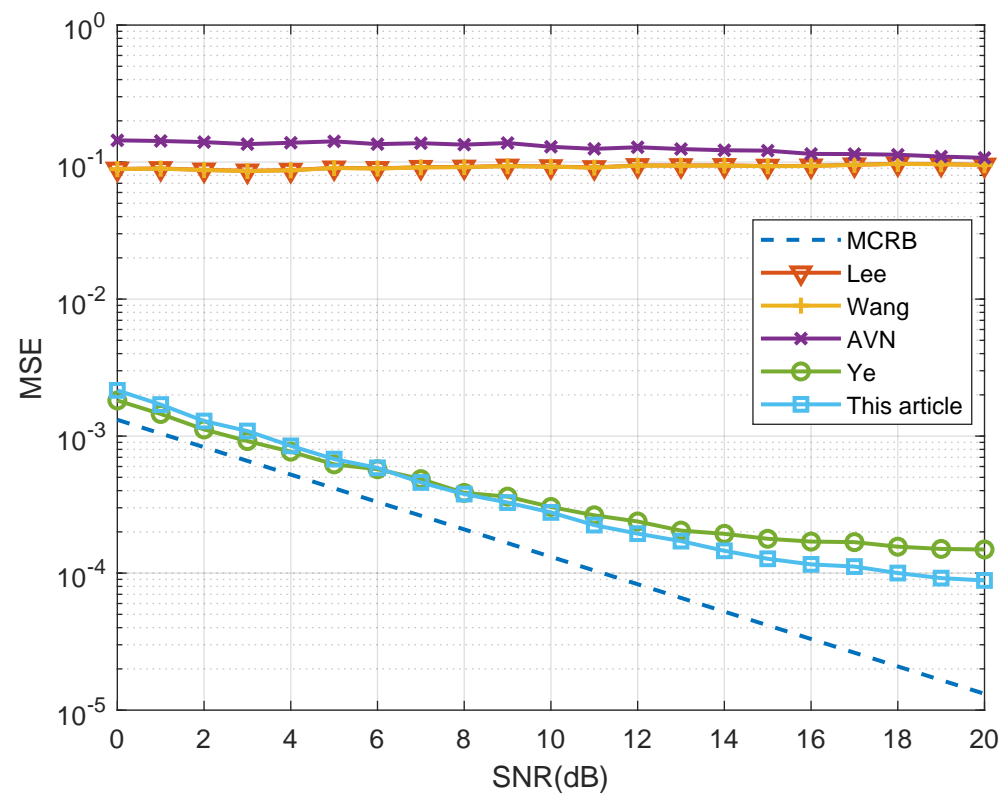

Figure 6. Timing error estimation algorithm used on OQPSK. 
Since Lee, Wang, and AVN do not work for OQPSK, in order to carry out a fair comparison, we also test OPSK signals in the following. Figure 7 shows the accuracy of the timing error estimation algorithms under different roll-off factors. Simulation conditions: adopt the QPSK signal; $N=108$; $L=12$; the frequency offset is zero. It can be seen from the figure that the performance of the Lee, Wang, and AVN algorithms is greatly affected by the roll-off factor, while the proposed algorithm and Ye algorithm are not sensitive to the roll-off factor. In addition, we can also see that the MSE performance of the proposed algorithm is slightly better than that of Ye and much better than that of Lee, Wang, and AVN.

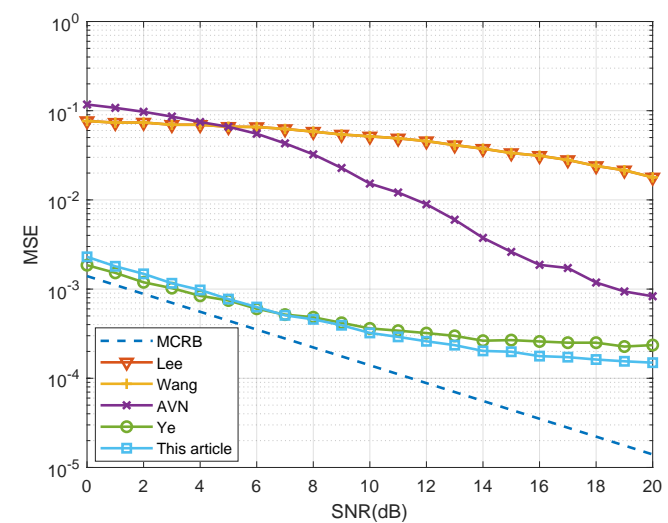

(a) The roll-off factor is 0.1

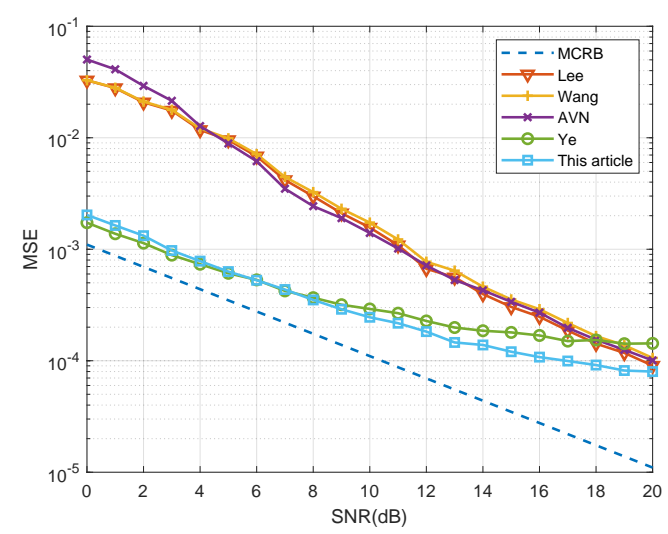

(c) The roll-off factor is 0.7

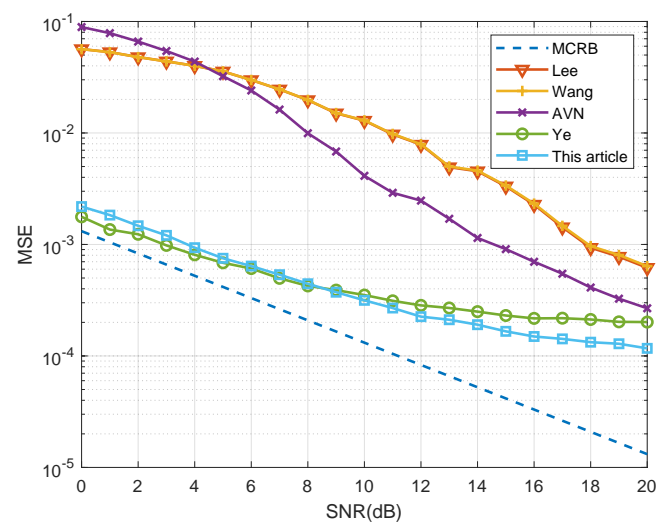

(b) The roll-off factor is 0.35

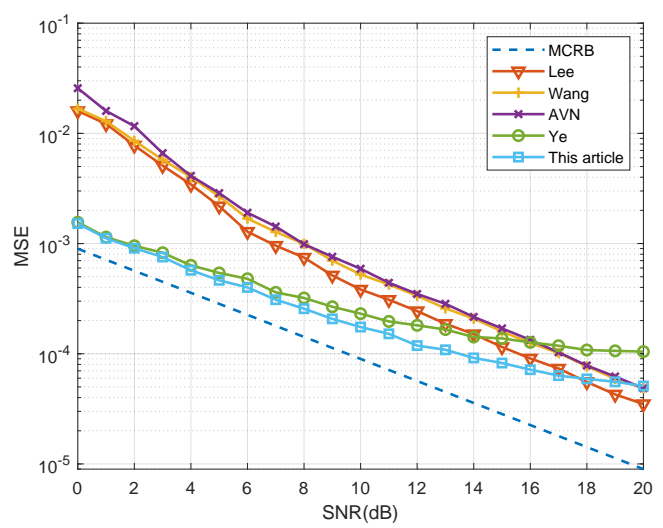

(d) The roll-off factor is one

Figure 7. Simulation of the timing error estimation algorithm under different roll-off factors.

If the roll-off factor is set to 0.35 , a normalized frequency offset of 0.05 is added, and the other conditions remain the same as those in Figure 7, then we can get the simulation in Figure 8. Figure 8 implies that with the existence of frequency offset, the Ye algorithm fails, while other algorithms are not affected.

From the above comparison, we can draw a conclusion: firstly, for the traditional Lee, Wang, and AVN algorithms, the performance is not only greatly affected by the roll-off factor, but also, they cannot be used on OQPSK signals. Secondly, although Ye solved these problems, it lost the ability of anti-frequency offset.

As we mentioned in Section 3.2.1, the anti-frequency offset ability in practical applications must be at least within the range of $(-0.1: 0.1)$. Figure 9 shows the anti-frequency offset ability of the algorithm in this article when it is used on OQPSK signals. Simulation conditions: $N=108 ; M=9 ; L=12$; the roll-off factor is 0.35 ; the added normalized frequency offsets is [-0.1:0.1] in steps of 0.025. It can be seen from the figure that as the frequency offset becomes larger, the estimation accuracy will slightly decrease, but 
overall, the difference is not large. Therefore, the timing algorithm in this article has good anti-frequency offset ability, and its range can fully meet the needs of the actual situation.

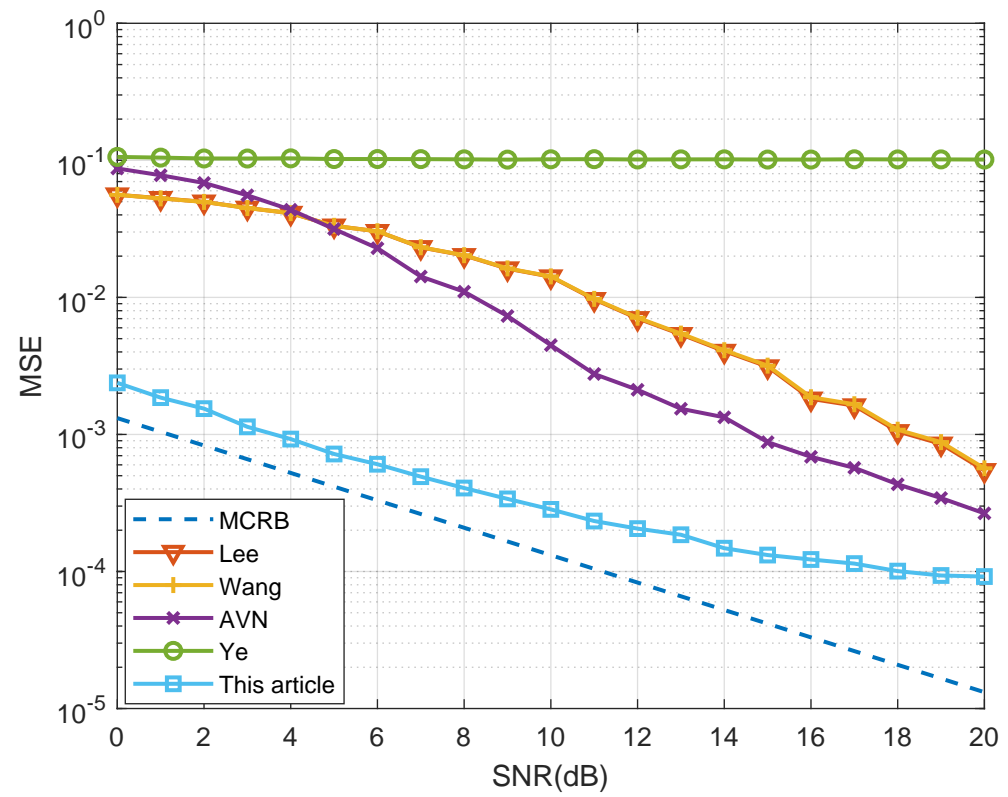

Figure 8. Timing error estimation simulation when adding 0.05 normalized frequency offset.

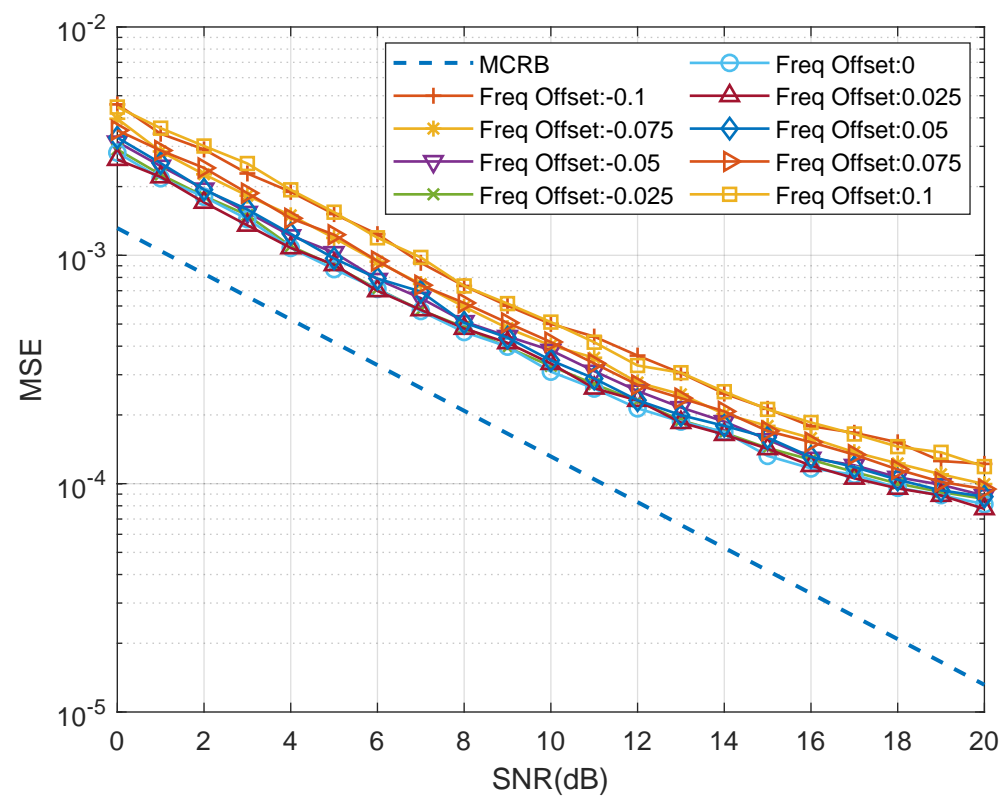

Figure 9. Timing estimation accuracy under different frequency offset.

All in all, the timing estimation algorithm in this article not only has high accuracy, but also, its performance is not affected by the roll-off factor; it also has a good ability of antifrequency offset. In addition, the value used in the interpolation of the timing estimation algorithm is generated during burst signal detection. According to Equation (29), it can be seen that the result can be achieved with only a very simple calculation.

\section{Conclusions}

In order to reduce the complexity of algorithm implementation, we propose an OQPSK synchronization parameter estimation algorithm based on burst signal detection. First of all, the burst signal detection is performed by adopting a simplified segment correlation algorithm, which greatly reduces the amount of calculation with a very small performance 
loss. After completing the burst signal detection, on the one hand, we use the results generated during the burst signal detection to estimate the frequency offset and then estimate the phase offset. On the other hand, the results of burst signal detection are also used to estimate the timing error. The simulation results show that the algorithm in this article not only greatly reduces the amount of calculation, but still retains good performance. More precisely, firstly, the estimation accuracy of the frequency offset estimation algorithm in this article is close to the L\&R and Fitz algorithms with a much wider estimation range; secondly, the timing estimation algorithm keeps high accuracy with excellent anti-frequency offset ability and is less sensitive to the change of the roll-off factor.

Author Contributions: Conceptualization, Z.L. and K.G.; methodology, Z.L., K.G., and K.Z.; software, Z.L.; validation, Z.L., K.G., and J.D.; writing, original draft preparation, Z.L.; writing, review and editing, Z.L., P.S., and L.D.; project administration, K.G., P.S., and J.D. All authors read and agreed to the published version of the manuscript.

Funding: This research was funded by the Development Program "Frontier Scientific and Technological Innovation" Special under Grant 2019QY0302, the National Natural Science Foundation of China under Grant U1736107, and the National Natural Science Foundation of China under Grant 61901417.

Institutional Review Board Statement: Not applicable.

Informed Consent Statement: Not applicable.

Data Availability Statement: The data presented in this study are available on request from the corresponding author.

Acknowledgments: We sincerely thank the members of the Communication Signal Analysis and Countermeasure Laboratory of the School of Information Engineering of Zhengzhou University and BeiBei Zhang for their help and support in developing this paper.

Conflicts of Interest: The authors declare no conflict of interest.

\section{References}

1. Zhang, N.N.; Xu, X.T.; Pang, J.C.; Liu, P. Service-Oriented Satellite Mobile Communication System Research on Effectiveness Evaluation. In Proceedings of the 2020 12th International Conference on Communication Software and Networks (ICCSN), Chongqing, China, 12-15 June 2020; pp. 95-99.

2. Min, S.; Joon, G.R.; Deock, G.O. On the network clock reference variation compensation for satellite TDMA network. In Proceedings of the 2017 International Conference on Information and Communication Technology Convergence (ICTC), Jeju, Korea, 18-20 October 2017; pp. 1216-1218.

3. Kulikov, G.V.; Nguyen, V.D.; Kulagin, V.P.; Lelyukh, A.A. Influence of synchronization errors on the noise immunity of receiving multi-position M-PSK and M-QAM signals. In Proceedings of the 2020 Systems of Signal Synchronization, Generating and Processing in Telecommunications (SYNCHROINFO), Svetlogorsk, Russia, 1-3 July 2020; pp. 1-5.

4. Oh, H.; Nam, J. Energy Detection Scheme in the Presence of Burst Signals. IEEE Signal Process. Lett. 2019, 26, 582-586. [CrossRef]

5. Zhang, J.; Xiang, J.C.; Zhu, Z.L. Unique code detection under low signal-to-noise ratio. J. Electron. Inf. Technol. 2001, 11, 1116-1122.

6. Choi, Z.Y.; Lee, Y.H. Frame synchronization in the presence of frequency offset. IEEE Trans. Commun. 2002, 50, 1062-1065. [CrossRef]

7. Zhang, P.Z. An improved algorithm for burst signal detection in TDMA system. Electron. Technol. Softw. Eng. 2016, 13, 40-41.

8. Yang, Z.H.; Yu, L.L.; Ding, Q.; Kang, S.J.; Han, M.J. Research on QPSK Carrier Synchronization Algorithm Based on Software Defined Radio. In Proceedings of the 2015 Third International Conference on Robot, Vision and Signal Processing (RVSP), Kaohsiung, Taiwan, 18-20 November 2015; pp. 276-279.

9. Meng, Z.K.; Zhang, H.L.; Wang, W.; Zhai, S.H. A Simplified Code-aided Carrier Synchronization Algorithm. In Proceedings of the International Conference on Safety Produce Informatization (IICSPI), Chongqing, China, 28-30 November 2019; pp. 283-286.

10. Xu, H.Z.; Zhu, H.; Zhu, S.F.; Yu, Z.Y. A wide range and low complexity carrier frequency offset estimation algorithm. Electron. J. 2019, 47, 2550-2555.

11. Lovell, B.C.; Williamson, R.C. The statistical performance of some instantaneous frequency estimators. IEEE Trans. Signal Process. 1992, 40, 1708-1723. [CrossRef]

12. Kay, S. A fast and accurate single frequency estimator. IEEE Trans. Acoust. Speech Signal Process. 1989, 37, 1987-1990. [CrossRef]

13. Luise, M.; Reggiannini, R. Carrier frequency recovery in all-digital modems for burst-mode transmissions. IEEE Trans. Commun. 1995, 43, 1169-1178. [CrossRef]

14. Fitz, M.P. Planar filtered techniques for burst mode carrier synchronization. IEEE Glob. Telecommun. Conf. 1991, 1, 365-369. 
15. Lee, S.J. A new non-data-aided feedforward symbol timing estimator using two samples per symbol. IEEE Commun. Lett. 2002, 6, 205-207.

16. Wang, Y.; Serpedin, E.; Ciblat, P. An alternative blind feedforward symbol timing estimator using two samples per symbol. IEEE Trans. Commun. 2003, 51, 1451-1455. [CrossRef]

17. Morelli, M.; D'Andrea, A.N.; Mengali, U. Feedforward ML-based timing estimation with PSK signals. IEEE Commun. Lett. 1997, 1, 82. [CrossRef]

18. Ye, Z.; Zhang, B.J.; Guo, D.X. Data aided forward bit timing estimation based on PN code. J. Signal Process. 2011, 27, 246-250.

19. APA Mengali, U.; D’Andrea, A.A.N. Synchronization Techniques for Digital Receivers; Plenum Press: New York, NY, USA, 1997; pp. 190-198.

20. Ascheid, G.; Meyr, H. Maximum Likelihood Detection and Synchronization by Parallel Digital Signal Processing. IEEE Glob. Commun. Conf. 1984, 2, 33.2.1-33.2.5. Available online: https://www.ice.rwth-aachen.de/publications/publication/Ascheid8 $4 \mathrm{a} /($ accessed on 1 January 2021)

21. Fu, D.; Willson, A.N.J. Trigonometric polynomial interpolation for timing recovery. IEEE Trans. Circuits Syst. I Regul. Pap. 2005, $52,338-349$. 